\title{
Increased expression of S100A6 promotes cell proliferation in gastric cancer cells
}

\author{
XIAO-HONG WANG ${ }^{1 *}$, HONG DU $^{2 *}$, LIN LI $^{2 *}$, DUAN-FANG SHAO ${ }^{2}$, XI-YAO ZHONG ${ }^{2}$, YING HU $^{1}$, \\ YI-QIANG LIU ${ }^{3}$, XIAO-FANG XING ${ }^{2}$, XIAO-JING CHENG ${ }^{2}$, TING GUO ${ }^{2}$, SHEN LI $^{2}$, ZI-YU LI$^{4}$, \\ ZHAO-DE BU ${ }^{4}$, XIAN-ZI WEN ${ }^{2}$, LIAN-HAI ZHANG ${ }^{4}$ and JIA-FU JI ${ }^{4}$ \\ ${ }^{1}$ Department of Tissue Bank, Key Laboratory of Carcinogenesis and Translational Research (Ministry of Education); \\ ${ }^{2}$ Gastrointestinal Cancer Translational Research Laboratory; Departments of ${ }^{3}$ Pathology and ${ }^{4}$ Surgery, \\ Peking University Cancer Hospital and Institute, Beijing 100142, P.R. China
}

Received July 3, 2015; Accepted October 26, 2016

DOI: $10.3892 / \mathrm{ol} .2016 .5419$

\begin{abstract}
S100A6 is involved in regulating the progression of cancer. S100A6 can regulate the dynamics of cytoskeletal constituents, cell growth and differentiation by interacting with binding or target proteins. The present study investigated whether S100A6 affects cell proliferation in gastric cancer cells by stimulating several downstream factors. Firstly, the expression and localization of S100A6 were investigated using immunohistochemical staining, an immunoelectron microscopy and laser confocal scanning. A ChIP-Chip assay was performed to determine the downstream factors of S100A6 using promoter Chip analysis, including approximately the -800 to +200 regions around the transcription starting point. Polymerase chain reaction analysis was performed to confirm this. It was found that the intensity of S100A6 staining was markedly higher in the cytoplasm and nucleus, and its expression level correlated with that of the Ki67 protein. The overexpression of S100A6 also promoted cell proliferation in AGS and BGC823 cell lines, detected using a Cell Counting-Kit 8 assay. In cells overexpressing S100A6, the expression levels of interleukin (IL)-8, cyclin-dependent kinase (CDK)5, CDK4, minichromosome maintenance complex component 7 (MCM7) and B-cell lymphoma 2 (Bcl2) were noticeably increased. In conclusion, the increased expression of S100A6 promoted cell proliferation by regulating the expression levels of IL-8, CDK5, CDK4, MCM7 and $\mathrm{Bcl} 2$ in gastric cancer cells.
\end{abstract}

Correspondence to: Dr Lian-Hai Zhang or Dr Jia-Fu Ji, Department of Surgery, Peking University Cancer Hospital and Institute, 52 Fucheng Road, Haidian, Beijing 100142, P.R. China

E-mail: zlhzlh@hotmail.com

E-mail: jiafuj@gmail.com

*Contributed equally

Key words: gastric cancer, S100A6, proliferation, ChIP-Chip

\section{Introduction}

S100A6 is a member of the S100 family, which is found localized to the cytoplasm and nucleus in a wide range of cell types $(1,2)$. Several studies have shown that S100A6 may be involved in the regulation of cancer progression (3). At present, the deregulated expression of S100A6 during malignant transformation has been described in human pancreatic cancer (4), malignant thyroid neoplasms (5), colorectal carcinoma (6,7), malignant melanoma (8), breast cancer (9), prostate cancer $(10,11)$ hepatocellular carcinoma (12) and renal cell carcinoma (13). Similar to other S100 proteins, S100A6 may promote cancer progression by specific involvement in cell survival and apoptotic pathways (14). In addition, S100A6 may interact with binding or target proteins, thereby regulating the dynamics of cytoskeletal constituents, cell growth and differentiation, and calcium homeostasis (1,14-17).

One possible mechanism underlying the effect of S100A6 in primary gastric cancer cells is in cell proliferation and DNA synthesis, which is supported by a previous study showing that the depletion of S100A6 in vascular endothelial cells increased the proportion of endothelial cells accumulating in the G2/M phase of the cell cycle (17). In addition, Joo et al (18) suggested that nuclear factor $(\mathrm{NF})-\mathrm{\kappa B}$ can regulate the gene expression of S100A6 in the HepG2 human hepatoblastoma cell line. Therefore, S100A6 may be one of the downstream factors of $\mathrm{NF}-\kappa \mathrm{B}$, which promotes cell-cycle progression. However, the precise mechanism of S100A6 as a key regulator of cell proliferation remains to be fully elucidated.

S100A6 is found localized to the nucleus in a wide range of cell types. ChIP-Chip (or ChIP-on-Chip), also known as genome-wide location analysis, is a technology used for isolating the genomic sites occupied by specific DNA binding proteins in living cells. This strategy can be used to annotate promoters in genomes by mapping the locations of the protein markers associated with these sites (19). The function of the eukaryotic promoter as an initiator for transcription is one of the most complex processes in molecular biology. These elements, including the TATA-box, GC-box, CAAT-box and the transcription start site, are known to function as binding sites for transcription factors and other proteins, which are 
involved in the initiation process. These promoter elements are present in various combinations separated by various distances in sequence.

In the present study, the expression and functional properties of S100A6, a major member of the S100 family, were investigated; primarily focusing on whether it affects cell proliferation in gastric cancer cells. The present study also investigated the downstream factors of S100A6.

\section{Materials and methods}

Patients and tissue specimens. In total, 196 patients with gastric cancer, including 132 males and 64 females (mean age, 57 years; age range, 26-80 years) were included in the present study and were diagnosed and surgically treated at Peking University Cancer Hospital (Beijing, China) between 1999 and 2007. Primary gastric carcinoma tissues and matched non-cancerous mucosal tissues were obtained from the patients and were fixed with $10 \%$ formaldehyde in PBS for immunohistochemistry. The investigations were performed following approval by the Ethics Committee of Peking University. General informed consent was obtained from each participant involved in the study.

Cell culture. The AGS and KATO 3 gastric cancer cell lines were obtained from American Type Culture Collection (Manassas, VA, USA). The BGC823 gastric cancer cell line was obtained from the Type Culture Collection of the Chinese Academy of Sciences (Shanghai, China). The cell lines were routinely grown in RPMI-1640 medium (Gibco; Thermo Fisher Scientific, Inc., Waltham, MA, USA), supplemented with $10 \%$ (v/v) fetal calf serum (FCS; Gibco; Thermo Fisher Scientific, Inc.) and antibiotics at $37^{\circ} \mathrm{C}$ in a humidified $5 \% \mathrm{CO}_{2}$ atmosphere.

Immunohistochemical analysis. Sections $(4 \mu \mathrm{m})$ of the formalin-fixed, paraffin-embedded tissues were mounted on poly-L-lysine-coated slides, deparaffinized in xylene, rehydrated with alcohol and rinsed with distilled water. Endogenous peroxidase activity was blocked with $3 \%$ hydrogen peroxide for $15 \mathrm{~min}$ at room temperature. Following heating the slides under pressure $\left(120^{\circ} \mathrm{C}\right.$ and $\left.103 \mathrm{kPa} / 15 \mathrm{psi}\right)$ in $10 \mathrm{mmol} / 1$ EDTA ( $\mathrm{pH} 8.0$ ) for $3 \mathrm{~min}$, the sections were incubated overnight at $4^{\circ} \mathrm{C}$ with mouse anti-S100A6 monoclonal antibody (1:500; cat. no. H00006277-M16; Abnova, Taipai, Taiwan), or mouse Ki-67 monoclonal antibody (1:100; cat. no. MS-1794-S0; LabVision, Fremont, CA, USA). Primary antibodies were detected using a two-step EnVision system (Dako, Glostrup, Denmark). As a negative control, the primary antibody was replaced with nonimmune mouse serum (Dako) to confirm its specificity.

Evaluation of slides. Evaluation of the S100A6 staining was performed using previously described scoring criteria (20). The recorded information included the subcellular location of the S100A6 staining (nuclear and/or cytoplasmic), the intensity of staining (negative, weak, moderate and strong) and the percentage of cells demonstrating positive immunoreactivity.

The reactivity of $\mathrm{Ki}-67$ was evaluated by counting the number of positive and negative tumor cell nuclei in at least four randomly selected fields in each section under a light microscope. The slides were visualized by two pathologists with no knowledge of the clinical data. Reproducibility of the scoring method between the two observers was $>90 \%$. In the remaining cases, if discrepancies were noted, the differences were settled through consensus following a review of the corresponding slides.

Laser confocal scanning. For double staining, mouse anti-human S100A6 antibody (1:500; Abnova) and rabbit anti-human Ki-67 antibody (1:100; cat. no. RM-9106-S0; LabVision) were used as the primary antibodies. The secondary antibodies used for double staining were fluorescein isothiocyanate (FITC)-conjugated goat anti-mouse antibody and rhodamine (TRITC)-conjugated goat anti-rabbit antibody (Jackson ImmunoResearch Laboratories, Inc., West Grove, PA, USA). Confocal images were captured using a Leica TCS SP5 confocal microscope (Leica Microsystems $\mathrm{GmbH}$, Mannheim, Germany). The excitation wavelength for TRITC was 586 and for FITC was $488 \mathrm{~nm}$. In addition, the nuclei of specimens were simultaneously stained with DAPI, with excitation at $358 \mathrm{~nm}$.

Immunoelectron microscopy. The post-embedding immunogold method was used to detect the S100A6 proteins. The anti-S100A6 antibody, obtained from Abnova, was used at a dilution of 1:40. The gastric cancer tissues $\left(1 \mathrm{~mm}^{3}\right.$ in size) were immersed for $6 \mathrm{~h}$ in fresh glutaraldehyde fixative solution, following which the tissues were washed in $0.2 \mathrm{M}$ sucrose and fixed in $1 \%$ osmium tetroxide for $4 \mathrm{~h}$ at $4^{\circ} \mathrm{C}$. Subsequently, the tissues were dehydrated using a graded acetone series $\left(-4^{\circ} \mathrm{C}\right)$, embedded in epoxy resin, and polymerized at $37^{\circ} \mathrm{C}$ for $24 \mathrm{~h}$ and $60^{\circ} \mathrm{C}$ for $48 \mathrm{~h}$. Ultrathin sections $(60 \mathrm{~nm})$ were obtained on an ultramicrotome and collected on nickel grids. All sections were incubated in $10 \% \mathrm{H}_{2} \mathrm{O}_{2}$, rinsed with phosphate-buffered saline enriched with $1 \%$ bovine serum albumin (Gibco; Thermo Fisher Scientific, Inc.) and $0.5 \%$ powdered skim milk three times ( 5 min each), and then incubated with the primary antibodies at $4^{\circ} \mathrm{C}$ overnight. Following incubation, the sections were washed three times in TBS ( $\mathrm{pH} 7.6)$, following which the sections were incubated with goat anti-mouse IgG antibody conjugated to $10 \mathrm{~nm}$ gold particles (Sigma-Aldrich; Merck Millipore, Darmstadt, Germany) at a dilution of 1:100 in TBS (pH 7.6). The sections were rinsed three times (10 min each) in enriched TBS, rinsed three times (10 min each) in distilled water, and then stained with uranyl acetate and lead citrate. The sections were visualized and images were captured using a Zeiss 900 transmission electron microscope (Zeiss $\mathrm{GmbH}$, Oberkochen, Germany).

Plasmids and S100A6 transfection. The cDNAs encoding human S100A6 were generated from human tissues using polymerase chain reaction (PCR) with the following forward and reverse primers containing $B a m \mathrm{HI}$ and XhoI sites: Forward 5'-AAGGATCCTACCGCTCCA AGCCCAGC-3' and reverse 5'-CTCGAGCGCC CTTGAGGGCTTCATTGT AGAT-3'. The PCR products were digested with BamHI and XhoI, and then directly subcloned into the BamHI and XhoI sites of pcDNA.3.1/myc-His C vectors (Invitrogen; Thermo Fisher Scientific, Inc.), which produce fusion proteins. 
The AGS and BGC823 human gastric cancer cell lines were maintained in high-glucose Dulbecco's modified Eagle's medium (Gibco; Thermo Fisher Scientific, Inc.) containing $10 \%$ FCS. At the time of transfection, the cells were $90 \%$ confluent. The cells in 6 -well plates $\left(8 \times 10^{5}\right)$ were transfected with a total of $4.0 \mu \mathrm{g}$ of plasmid DNAs using Lipofectamine $^{\mathrm{TM}} 2000$ reagent (Invitrogen; Thermo Fisher Scientific, Inc.). The medium was replaced $6 \mathrm{~h}$ later. The cells were incubated at $37^{\circ} \mathrm{C}$ in a $\mathrm{CO}_{2}$ incubator for $48 \mathrm{~h}$ prior to assessment of transgene expression. G418 (300 ng/ml; Gibco; Thermo Fisher Scientific, Inc.) was used to screen and isolate the resistant colonies. The efficiency of stable transfection was confirmed using PCR and western blot analyses.

Reverse transcription-quantitative PCR (RT-qPCR) analysis. RT-qPCR was performed to confirm successful transfection of the cell lines with S100A6 and to determine the expression levels of interleukin (IL)-8, IL-2, IL-13, IL-6R, cyclin-dependent kinase (CDK) 4, CDK5, minichromosome maintenance complex component 7 (MCM7), B-cell lymphoma 2 ( $\mathrm{Bcl} 2)$ and MYC. Total RNA was extracted using TRIzol reagent (Invitrogen; Thermo Fisher Scientific, Inc.), according to the manufacturer's protocol. Total RNA $(1 \mu \mathrm{g})$ was reverse transcribed using the GoScript ${ }^{\mathrm{TM}}$ Reverse Transcription System (Promega Corporation, Madison, WI, USA). qPCR was performed using the SYBR Green PCR Master Mix kit (Applied Biosystems; Thermo Fisher Scientific, Inc.) with the following cycling conditions: $95^{\circ} \mathrm{C}$ for $10 \mathrm{~min}$, followed by 40 cycles at $95^{\circ} \mathrm{C}$ for $15 \mathrm{sec}, 60^{\circ} \mathrm{C}$ for $10 \mathrm{sec}$ and $72^{\circ} \mathrm{C}$ for $60 \mathrm{sec}$. Each sample was run thrice. The primer sequences were as follows: S100A6 forward, 5'-AAGCTGCAGGATGCT GAAAT-3' and reverse, 5'-CCCTTGAGGGCTTCATTGTA-3'; IL-8 forward, 5'-ACTGAGAGTGATTGAGAGTGGAC-3' and reverse, 5'-AACCCTCTGCACCCAGTTTTC-3'; IL-2 forward, 5'-AACTCCTGTCTTGCATTGCAC-3' and reverse, 5'-GCTCCAGTTGTAGCTGTGTTT-3'; IL-13 forward, 5'-CCTCATGGCGCTTTTGTTGAC-3' and reverse, 5'-TCT GGTTCTGGGTGATGTTGA-3'; IL-6R forward, 5'-CCCCTC AGCAATGTTGTTTGT-3' and reverse, 5'-CTCCGGGAC TGCTAACTGG-3'; CDK4 forward, 5'-ATGGCTACCTCT CGATATGAGC-3' and reverse, 5'-CATTGGGGACTCTCA CACTCT-3'; CDK5 forward, 5'-GGAAGGCACCTACGG AACTG-3' and reverse, 5'-GGCACACCCTCATCATCGT-3'; MCM7 forward, 5'-CCTACCAGCCGATCCAGTCT-3' and reverse, 5'-CCTCCTGAGCGGTTGGTTT-3'; Bcl2 forward, 5'-GGTGGGGTCATGTGTGTGG-3' and reverse 5'-CGG TTCAGGTACTCAGTCATCC-3'; MYC forward, 5'-GGC TCCTGGCAAAAGGTCA-3' and reverse, 5'-CTGCGTAGT TGTGCTGATGT-3'; and $\beta$-actin forward, 5'-AAATCTGGC ACCACACCTTC-3' and reverse, 5'-GGGGTGTTGAAGGTC TCAAA-3'. The expression of each gene was determined as the ratio of the mRNA expression of each target gene to that of $\beta$-actin, using the $2^{-\Delta \Delta \mathrm{Cq}}$ method (21).

Western blot analysis. Total proteins were extracted using TRIzol reagent, according to the manufacturer's protocol. Protein concentration was determined using a Bicinchoninic Acid Protein Assay kit (Thermo Fisher Scientific, Inc.). Samples were denatured in SDS sample loading buffer by heating at $100^{\circ} \mathrm{C}$ for $3 \mathrm{~min}$ and resolved by $12 \%$ SDS-PAGE, followed by transfer to polyvinylidene difluoride membranes. Blots were blocked with 5\% nonfat milk in PBS for 30 min and then incubated with the mouse anti-S100A6 monoclonal antibody $(1: 2,000)$ and mouse anti-tubulin monoclonal antibody (1:5,000; cat. no. T5168; Sigma-Aldrich; Merck Millipore) for $2 \mathrm{~h}$ at room temperature. After washing, the membranes were incubated with the horseradish peroxidase-conjugated anti-mouse antibody (1:1,000; cat. no. sc-2005; Santa Cruz Biotechnology, Inc., Santa Cruz, CA, USA). Protein-antibody complexes were visualized using the SuperSignal West Pico Chemiluminescent Substrate (Thermo Fisher Scientific, Inc.).

Cell proliferation assay. The BGC823, AGS, BGC823 cells transfected with pcDNA.3.1/myc-His C plasmid (BGC823/control), AGS cells transfected with pcDNA.3.1/myc-His C plasmid (AGS/control), BGC823 cells transfected with S100A6/pcDNA.3.1/myc-His C plasmid (BGC823/S100A6) and AGS cells transfected with S100A6/pcDNA.3.1/myc-His C plasmid (AGS/S100A6) were digested with trypsin-EDTA buffer (Gibco; Thermo Fisher Scientific, Inc.). A suspension of $2 \times 10^{3}$ cells $/ 90 \mu 1$ medium was added to each well of 96-well plates and allowed to grow; each concentration had three duplicate wells. The plates were incubated for $1,2,3,4,5$ and 6 days following which $10 \mu 1$ of Cell Counting Kit-8 (CCK8) solution (Dojindo Molecular Technologies, Inc., Kumamoto, Japan) was added to each well and the plates were incubated for another $2 \mathrm{~h}$ at $37^{\circ} \mathrm{C}$. Subsequently, the absorbance was read at $450 \mathrm{~nm}$ using a Model 680 microplate reader (Bio-Rad Laboratories, Inc., Hercules, CA, USA) (22).

ChIP-Chip assay. KATO3 cells $(10,000,000)$ were prepared, crosslinked with $1 \%$ formaldehyde and lysed with lysate buffer. Sonication was used to break genomic DNA into small DNA fragments. Anti-S100A6 (Abnova) was added and used to immunoprecipitate DNA fragments corresponding to the promoter regions. IgG was used as a control. The DNA fragments bound by proteins were then isolated, and DNA was hybridized to the GLAS-H20K-Chip (Aviva Systems Biology, Corp., San Diego, CA, USA), with the transcription starting point approximately in the -800 to +200 regions. The microarray was then scanned, and two images corresponding to anti-S100A6 and IgG (control) were captured. Subsequently, the ratio between anti-S100A6 and $\mathrm{IgG}$ was calculated. The factors for which the ratio was $>2$ were selected. Gene Ontology (GO) analysis was then performed to clarify the gene function (http://geneontology.org).

Statistical analysis. Spearman's rank correlation was performed to analyze the correlation between the mRNA expression of S100A6 and Ki67. Two-way analysis of variance was performed to analyze the results of the CCK8 assay. $\mathrm{P}<0.05$ was considered to indicate a statistically significant difference and all tests were two-tailed. The statistical analysis was performed using SPSS V16.0 software (SPSS, Inc., Chicago, IL, USA).

\section{Results}

Expression of S100A6 and its localization in gastric cancer tissues. A comparison between primary gastric carcinoma 
A

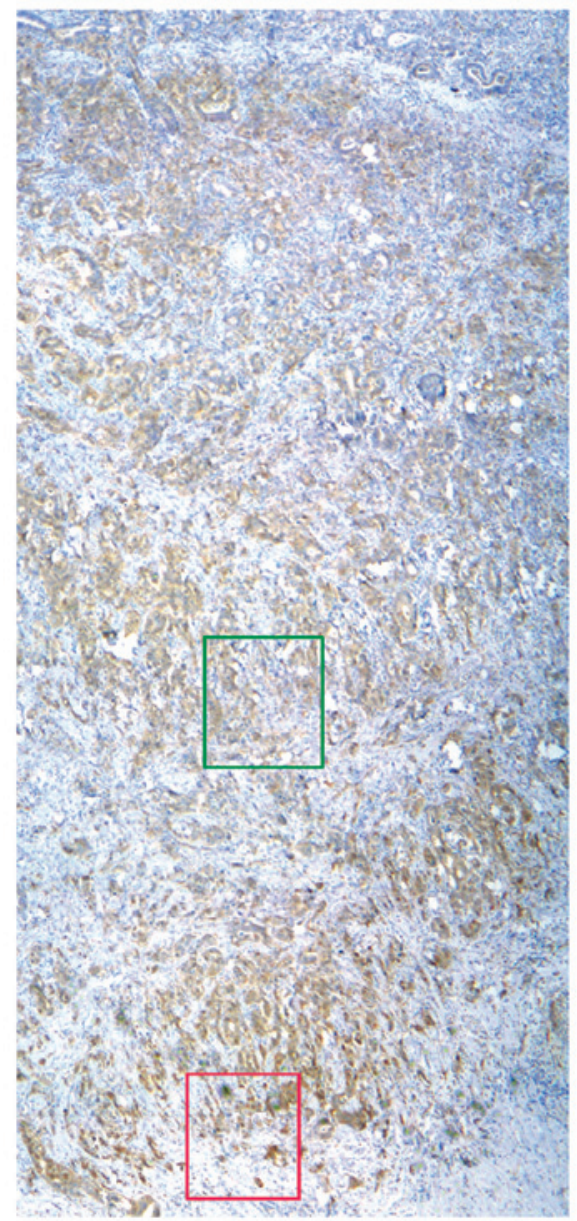

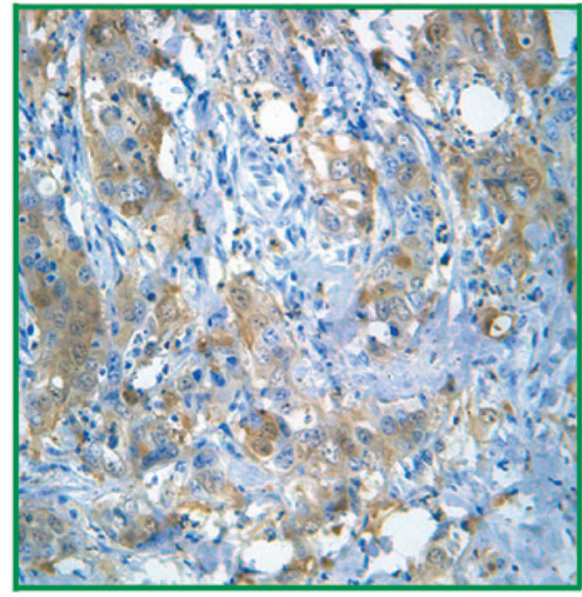

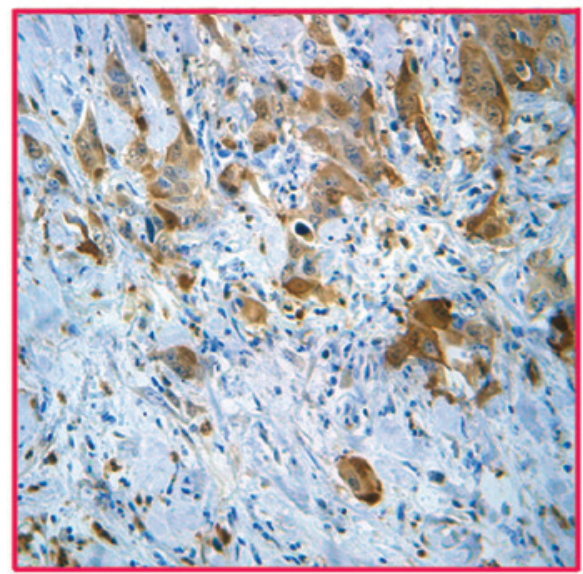

B

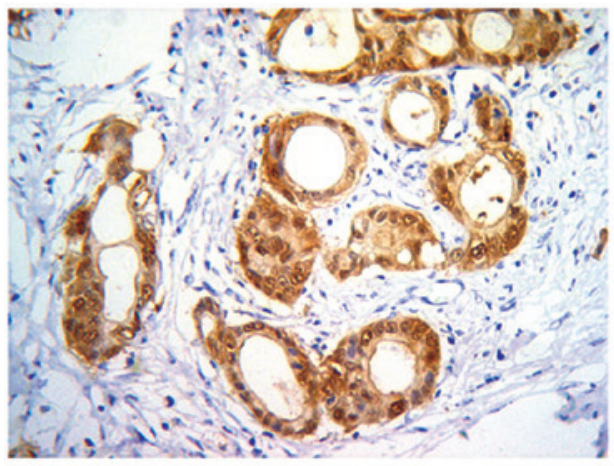

Cytoplasm+ / Nucleus+

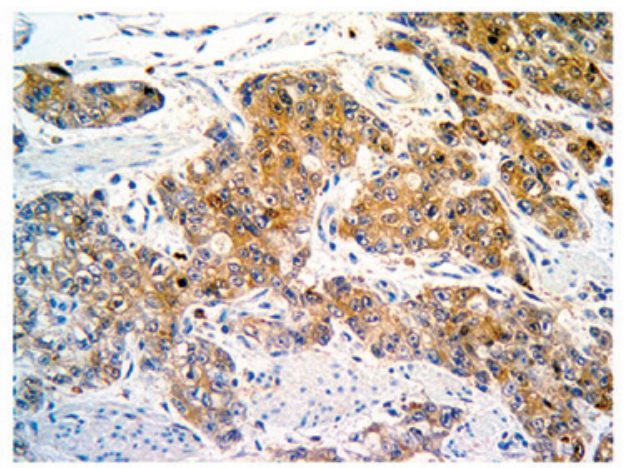

Cytoplasm+ / Nucleus -

Figure 1. Expression of S100A6 in primary gastric carcinoma, detected using immunohistochemical staining. (A) Image shows marked staining of S100A6 in the tumor (magnification, x40). Higher magnification images to the right show S100A6 staining in the (top) central region of the tumor and (bottom) invasion in front of the primary tumor tissues (B) Representative images of the expression of S100A6 (cytoplasm ${ }^{+} /$nucleus $^{+}$; cytoplasm $^{+} /$nucleus $^{-}$).

tissues and matched non-cancerous mucosal tissues revealed significant differences in the protein levels of S100A6 in these tissues. In the primary gastric carcinoma tissues, the intensity of S100A6 staining was markedly higher in the cytoplasm and nucleus of primary gastric carcinoma tissues, compared with the matched non-cancerous mucosa, which had either no or weak cytoplasmic staining. The S100A6 nuclear staining was significantly higher in the invading fronts with structural atypia, compared with the central portions with glandular structures (Fig. 1A and B). The ultrastructural images captured using an immunoelectron microscope showed that S100A6 was deposited within the cytoplasm and nucleus, whereas it was distributed in the nucleoplasmic and nucleolar structures in gastric cancer cells (Fig. 2A).

Expression of S100A6 is associated with the Ki-67 labeling index. To investigate whether the expression of S100A6 was associated with in vivo cell proliferation, 108 samples underwent Ki-67 and S100A6 immunohistochemical staining, respectively. Statistical analysis revealed a positive correlation between high expression of S100A6 and a high Ki-67 labeling index ( $\mathrm{P}=0.043$; Fig. 2B and $\mathrm{C})$. In total, 10 representative slides 
A

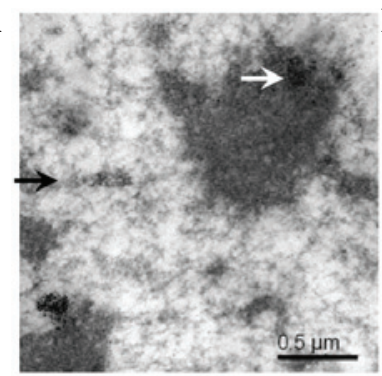

B

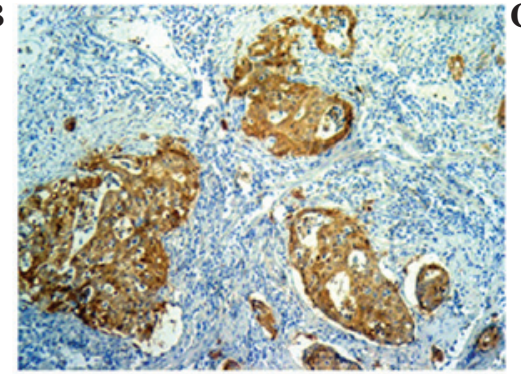

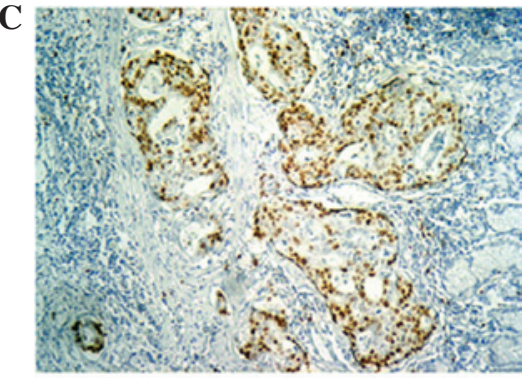

D
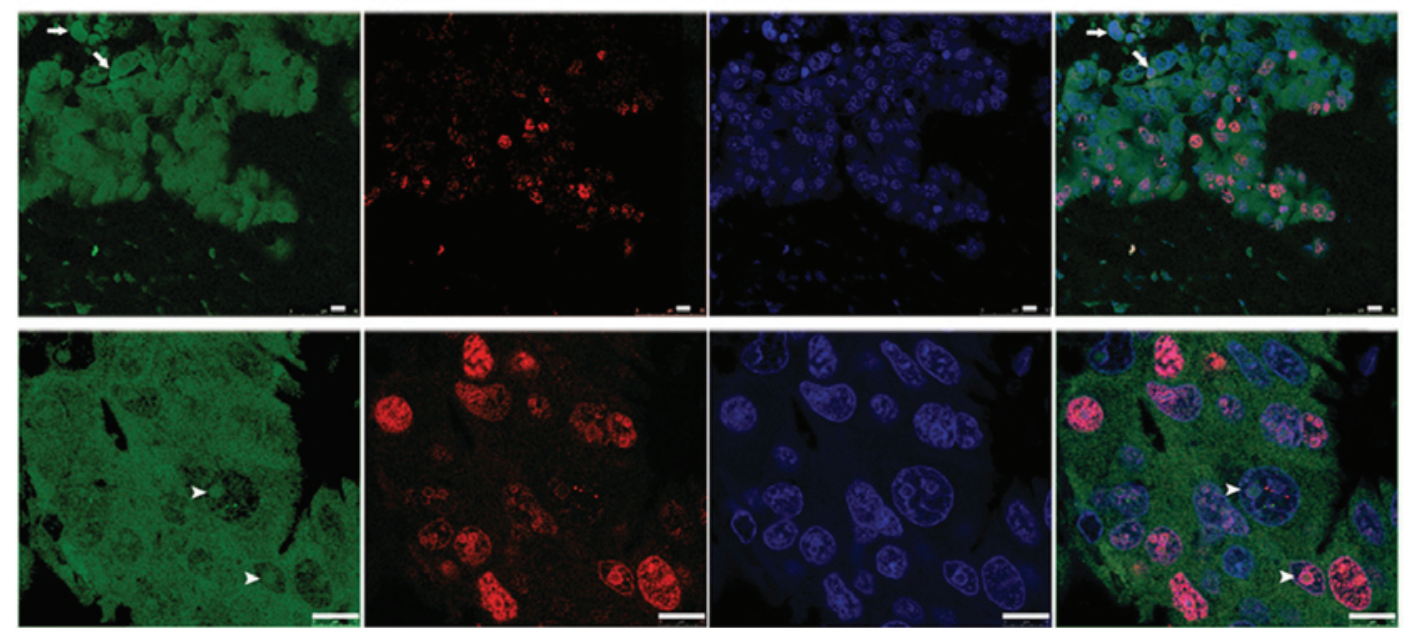

Ki-67

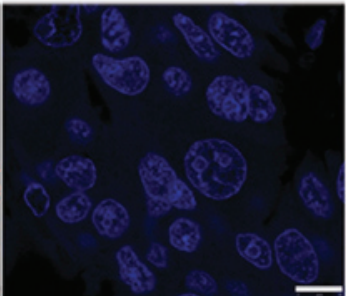

DAPI

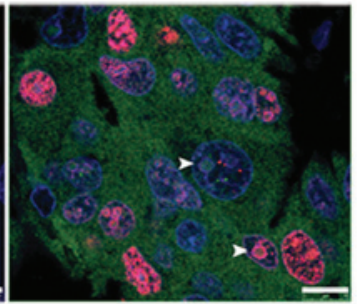

Merge

Figure 2. Localization of S100A6 and the association between the expression of S100A6 and the Ki-67 antigen. (A) Ultrastructural localization of the expression of S100A6 in gastric cancer tissue, detected using immunoelectron microscopy: The cancer cells exhibit marked immunoreactivity, as indicated by gold labeling particles within the nucleus, including the nucleoplasm and nucleolus (indicated by the black arrow). Scale bar=0.5 $\mu \mathrm{m}$. (B) S100A6-positive stained cancer cells (magnification, x100). (C) Ki-67 was positive in the same sample (magnification, x100), according to immunohistochemistry. (D) Immunofluorescence images captured by confocal laser scanning. Ki-67 (red; rhodamine-conjugated) was predominantly localized to the nucleus of cancer cells within the same positive areas of S100A6 (green; fluorescein isothiocyanate-conjugated). S100A6 subcellular localization included the cytoplasm (white arrows), whole nucleus and nucleolus (white arrowheads). Scale bar=10 $\mu \mathrm{m}$. Nu, nucleus; nus, nucleolus.

were selected for immunofluorescence staining and consecutive laser confocal scanning, which showed that the Ki-67 antigen was found localized to the nuclear region (Fig. 2D).

Effect of S100A6 transfection on cell proliferation. The correlation between S100A6 and cell proliferation was also investigated in vitro. Successful transfection of AGS and BGC823 cells with S100A6 was confirmed by western blotting (Fig. 3A and B). The present study examined whether S100A6 transfection promoted cell proliferation. The AGS, AGS/control, AGS/S100A6, BGC823, BGC823/control and BGC823/S100A6 cells were incubated for 1, 2, 3, 4, 5 and 6 days, and the growth rates were calculated using a CCK8 assay. The results suggested that the cell proliferation rate increased gradually and reached its highest level following 6 days of incubation. The proliferative abilities of the AGS/S100A6 and BGC823/S100A6 cells were markedly increased compared with those of the controls (Fig. 3C and D).

S100A6 combines with the gene promoter region and promotes the expression of the downstream cancer-associated genes. Based on the above results, it was concluded that S100A6 was located at nucleolar structures and that it regulated cell proliferation. Subsequently, a ChIP-Chip assay was performed, with the promoter Chip including approximately
-800 to +200 regions around the transcription starting point. The results showed the involvement of 1,328 genes, which regulate metabolic process, cell proliferation, cell cycle and cell apoptosis. GO category analysis suggested that 57 factors were associated with cell proliferation (Table I). In addition, the common sites of the promoter regions of the 1,328 involved genes were analyzed using the human and mouse promoter database $(-100$ to +1$)$. The results showed that the promoter regions of the majority of genes had SP1 protein binding sites, for example the cartilage oligomeric matrix protein gene (Fig. 4A and B).

To confirm the effect of S100A6 on the expression of factors associated with the malignant cell phenotype, the present study compared the expression levels of IL-8, IL-2, IL-13, IL-6R, CDK4, CDK5, MCM7, Bcl2 and MYC prior to and following S100A6 transfection of the BGC823 and AGS cell lines. RT-qPCR analysis was performed, which revealed that, following S100A6 transfection in the AGS cell lines, the mRNA expression levels of IL-8, CDK5, CDK4, MCM7 and $\mathrm{Bcl} 2$ were increased (Fig. 5A-E, respectively).

\section{Discussion}

S100A6 is a member of the S100 family of proteins, which are found localized to the cytoplasm and nucleus in a wide range of 
Table I. Factors associated with cell proliferation detected using the ChIP-Chip assay.

\begin{tabular}{|c|c|c|c|}
\hline Accession no. & Name & Ratio $\mathrm{Ab} / \mathrm{IgG}$ & Description \\
\hline NM_007037 & ADAMTS8 & 9.76 & ADAM metallopeptidase with thrombospondin type 1 motif, 8 \\
\hline NM_000584 & IL-8 & 9.44 & Interleukin-8 \\
\hline NM_000075 & CDK4 & 9.25 & Cyclin-dependent kinase 4 \\
\hline NM_005962 & MXI1 & 7.66 & MAX interactor 1 \\
\hline NM_145080 & NSE1 & 5.45 & Non-SMC element 1 homolog (S. cerevisiae) \\
\hline NM_001352 & DBP & 4.67 & Group-specific component (vitamin D binding protein) \\
\hline NM_145307 & PLEKHK1 & 4.62 & Pleckstrin homology domain containing, family K member 1 \\
\hline NM_002188 & IL-13 & 4.48 & Interleukin-13 \\
\hline NM_002415 & MIF & 4.13 & S100 calcium binding protein A9 (calgranulin B) \\
\hline NM_002211 & ITGB1 & 4.03 & $\begin{array}{l}\text { Integrin, } \beta 1 \text { (fibronectin receptor, } \beta \text { polypeptide, antigen CD29 includes } \\
\text { MDF2, MSK12) }\end{array}$ \\
\hline NM_001282 & AP2B1 & 3.92 & Adaptor-related protein complex $2, \beta 1$ subunit \\
\hline NM_006191 & PA2G4 & 3.88 & Proliferation-associated 2G4, $38 \mathrm{kDa}$ \\
\hline NM_014366 & NS & 3.82 & Guanine nucleotide binding protein-like 3 (nucleolar) \\
\hline NM_000565 & IL-6R & 3.55 & Interleukin-6 receptor \\
\hline NM_003711 & PPAP2A & 3.51 & Phosphatidic acid phosphatase type $2 \mathrm{~A}$ \\
\hline NM_002457 & MUC2 & 3.45 & Mucin 2, oligomeric mucus/gel-forming \\
\hline NM_001565 & CXCL10 & 3.29 & Chemokine (C-X-C motif) ligand 10 \\
\hline NM_005880 & DNAJA2 & 3.25 & DnaJ (Hsp40) homolog, subfamily A, member 2 \\
\hline NM_000369 & TSHR & 3.21 & Thyroid stimulating hormone receptor \\
\hline NM_000633 & BCL2 & 3.18 & B-cell CLL/lymphoma 2 \\
\hline NM_002825 & PTN & 3.15 & Pleiotrophin (heparin binding growth factor 8 , neurite growth-promoting factor 1 ) \\
\hline NM_153632 & HOXA3 & 3.14 & Homeobox A3 \\
\hline NM_182776 & MCM7 & 3.11 & MCM7 minichromosome maintenance deficient 7 (S. cerevisiae) \\
\hline NM_002355 & M6PR & 3.10 & Mannose-6-phosphate receptor (cation dependent) \\
\hline NM_004935 & CDK5 & 2.94 & Cyclin-dependent kinase 5 \\
\hline NM_006510 & RFP & 2.93 & Ret finger protein \\
\hline NM_000595 & LTA & 2.90 & Lymphotoxin $\alpha$ (TNF superfamily, member 1$)$ \\
\hline NM_000586 & IL-2 & 2.86 & Interleukin-2 \\
\hline NM_005438 & FOSL1 & 2.86 & FOS-like antigen 1 \\
\hline NM_005524 & HES1 & 2.81 & Hairy and enhancer of split 1, (Drosophila) \\
\hline NM_198218 & ING1 & 2.79 & Inhibitor of growth family, member 1 \\
\hline NM_003254 & TIMP1 & 2.72 & TIMP metallopeptidase inhibitor 1 \\
\hline NM_005542 & INSIG1 & 2.69 & Insulin induced gene 1 \\
\hline NM_004236 & TRIP15 & 2.62 & COP9 constitutive photomorphogenic homolog subunit 2 (Arabidopsis) \\
\hline NM_016041 & F-LANa & 2.54 & Der1-like domain family, member 2 \\
\hline NM_001311 & CRIP1 & 2.50 & Cysteine-rich protein 1 (intestinal) \\
\hline NM_020310 & MNT & 2.49 & MAX binding protein \\
\hline NM_003255 & TIMP2 & 2.46 & TIMP metallopeptidase inhibitor 2 \\
\hline NM_001233 & CAV2 & 2.45 & Caveolin 2 \\
\hline NM_173176 & PTK2B & 2.42 & PTK2B protein tyrosine kinase $2 \beta$ \\
\hline NM_000306 & POU1F1 & 2.40 & POU domain, class 1 , transcription factor 1 (Pit1, growth hormone factor 1$)$ \\
\hline NM_006034 & TP53I11 & 2.40 & Tumor protein p53 inducible protein 11 \\
\hline NM_015902 & DD5 & 2.39 & E3 ubiquitin protein ligase, HECT domain containing, 1 \\
\hline NM_001981 & EPS 15 & 2.35 & Epidermal growth factor receptor pathway substrate 15 \\
\hline NM_004429 & EFNB1 & 2.35 & Ephrin-B1 \\
\hline NM_015066 & TRIM35 & 2.33 & Tripartite motif-containing 35 \\
\hline NM_004356 & CD81 & 2.31 & CD81 molecule \\
\hline NM_000508 & FGA & 2.26 & Fibrinogen $\alpha$ chain \\
\hline NM_005378 & MYCN & 2.24 & v-myc myelocytomatosis viral related oncogene, neuroblastoma derived (avian) \\
\hline NM_006763 & BTG2 & 2.24 & BTG family, member 2 \\
\hline NM_006763 & BTG2 & 2.24 & BTG family, member 2 \\
\hline
\end{tabular}


Table I. Continued.

\begin{tabular}{llcl}
\hline Accession no. & Name & Ratio Ab/IgG & \multicolumn{1}{c}{ Description } \\
\hline NM_006292 & TSG101 & 2.11 & Tumor susceptibility gene 101 \\
NM_006806 & BTG3 & 2.09 & BTG family, member 3 \\
NM_033224 & PURB & 2.05 & Purine-rich element binding protein B \\
NM_000194 & HPRT1 & 2.02 & Hypoxanthine phosphoribosyltransferase 1 (Lesch-Nyhan syndrome) \\
NM_002467 & MYC & 2.02 & Nucleolar protein 3 (apoptosis repressor with CARD domain) \\
NM_153719 & NUP62 & 2.00 & Nucleoporin 62 kDa
\end{tabular}

A

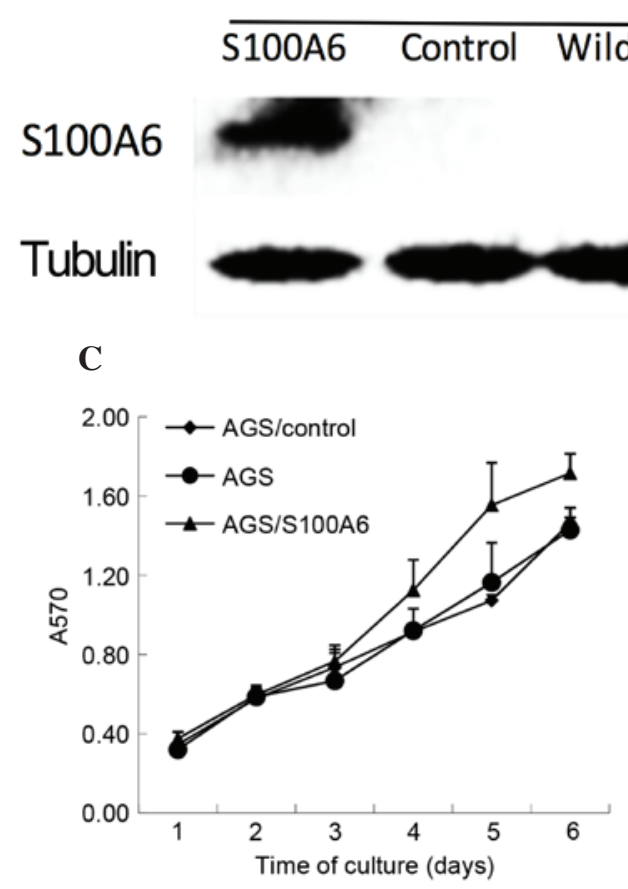

B

S100A6 Control Wild type

Figure 3. S100A6 overexpression increases the proliferation in AGS and BGC823 cells. Ectopic expression of S100A6 in (A) AGS and (B) BGC823 cells at the protein level was confirmed by western blot analysis. The effect of S100A6 overexpression on the cell proliferation of (C) AGS cells and (D) BGC823 cells was evaluated by cell counting kit-8 assays.

cell types. S100A6 is identified based on its cell cycle-dependent expression, as it is preferentially expressed in the $G_{1}$ phase of the cell cycle following mitogenic stimuli. It has previously been suggested that the expression of S100A6 is associated with cell proliferation; it is expressed in several types of cancer phenotypes. S100A6 is implicated in the regulation of different cellular processes, including cell cycle progression, differentiation and apoptosis. However, the precise biological or cellular functions of S100A6 remain to be fully elucidated, and contradictory roles have been suggested (23).

Localization experiments suggested that S100A6 was present in the cytoplasm and nucleus, where it distributed to nucleoplasmic and nucleolar structures in the gastric cancer cells, and that immunostaining was significantly higher in the invading fronts. It is possible that the S100A6 protein performs multiple functions in the cytoplasm and nucleus of primary gastric cancer cells.

To further investigate the role of S100A6 in gastric cancer, the S100A6 gene was transfected into the AGS and
BGC823 gastric cancer cell lines. The results of the CCK8 assay suggested that overexpression of S100A6 promoted cell proliferation. Thus, S100A6 affected cell proliferation in the AGS and BGC823 cell lines. In addition, a positive correlation between high expression levels of S100A6 and a high Ki-67 labeling index was found in vivo. These results confirmed that S100A6 was associated with the proliferation of gastric cancer cells.

Subsequently, the present study investigated how S100A6 affects cell proliferation. Examination of the expression of S100A6 in the gastric cancer cell lines revealed that the KATO3 cells had high levels of S100A6 in the cytoplasm and nucleus. Therefore, the KATO3 cells were selected to perform a ChIP-Chip assay. The ChIP-Chip assay was used to detect the gene promoter regions to which S100A6 may bind directly or indirectly. The ChIP-Chip results suggested that S100A6 may regulate the expression of 1,328 factors. Analyses of the GO categories included several genes, which are relevant to cell proliferation, cell migration and invasion. As evidence has 
A

COMP gene promoter:

$>$ COMP : chr19 : 18747325 [-100..1] (-) [human, homo sapiens] ctgtttaccttgaggetggacgttgggcagggetgtggtgggccgtccct ggggccggccgtgccttggggataaataggecccgcgggcctcgtgggcg gt

B

Binding sites prediction:

Sequence COMPchr1918747325-100..1-humanHomosapiens

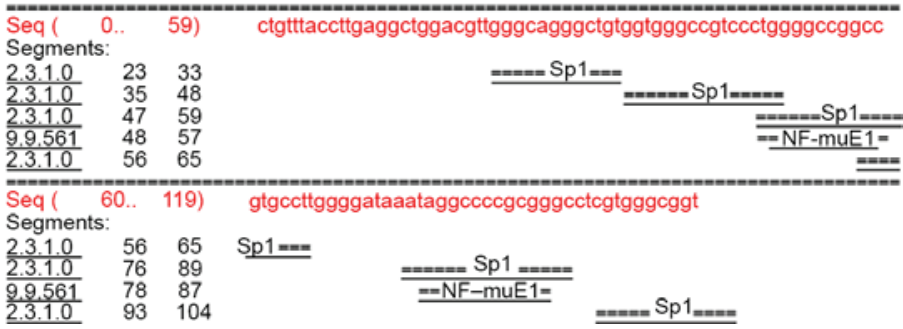

Figure 4. Common sites identified in promoter region analysis. (A) COMP gene promoter; (B) protein binding site predictions. COMP, cartilage oligomeric matrix protein.

A

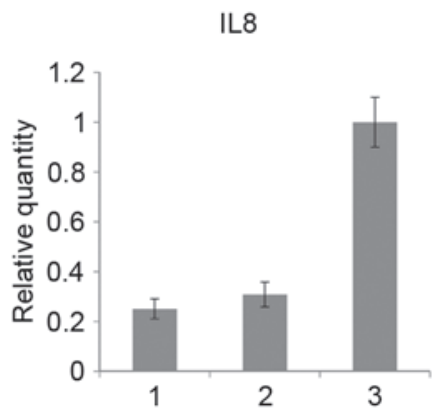

B

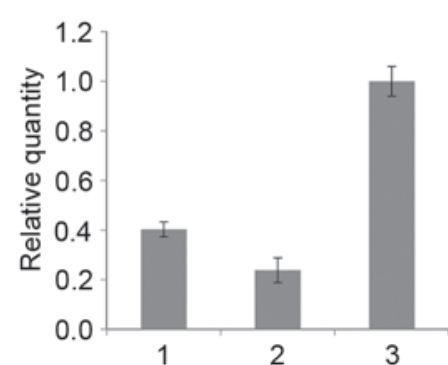

C

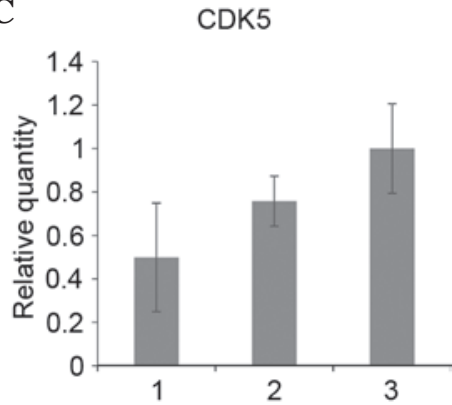

D

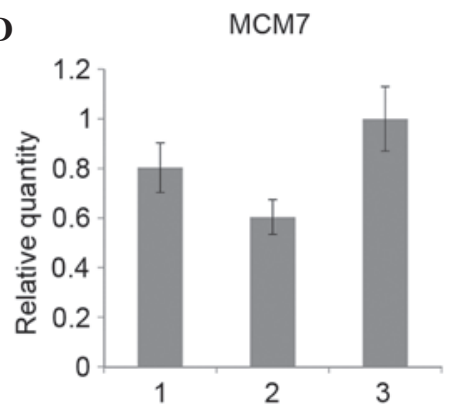

E

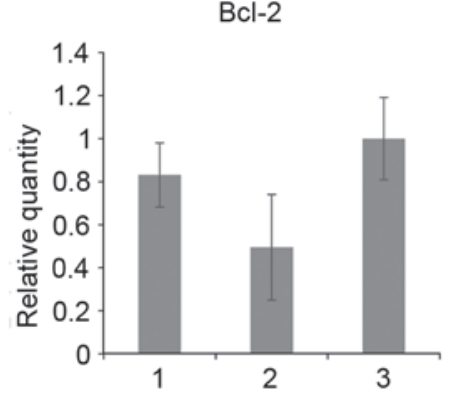

Figure 5. Alterations in the expression levels of downstream genes prior to and following S100A6 transfection. (A) IL8, (B) CDK4, (C) CDK5, (D) MCM7, and (E) Bcl-2. 1, AGS cells; 2, AGS/control cells; 3, AGS/S100A6 cells; IL-8, interleukin-8; CDK, cyclin-dependent kinase; MCM7, minichromosome maintenance complex component 7; Bcl2, B-cell lymphoma 2.

suggested that S100A6 regulates the levels of certain genes involved in cell proliferation, including IL-8 (24), IL-2 (25), IL-13 (26), IL-6R (27), CDK4 (28), CDK5 (29), MCM7 (30), $\mathrm{Bcl} 2$ (29) and MYC (31), these genes were selectively assessed. To confirm and to evaluate their expression levels, mRNA quantification was performed. The overexpression of S100A6 caused a marked increase in the mRNA levels of CDK4, CDK5, IL-8, Bcl2 and MCM7. This overexpression is a consequence of the specific activation of the CDK4, CDK5, IL-8, Bcl2 and MCM7 gene promoters by S100A6, as demonstrated by the ChIP-Chip assay. Thus, S100A6 may affect the malignant cell phenotype by regulating the expression levels of CDK4, CDK5, IL-8, Bcl2 and MCM7.

The common sites of the promoter region in the 1,328 involved genes were predicted using the human and mouse promoter database $(-100$ to +1$)$. The results showed that the promoter regions of the majority of genes had SP1 protein binding sites. The determination of how S100A6 binds to the gene promoters warrants further investigation. Previous reports have suggested that there is no DNA-binding domain in the S100A6 protein. S100A6 interacts with p53 in the presence of calcium ion affinity chromatography and co-immunoprecipitation (32). p53 can mediate the promoter attenuation of the cyclin B1 promoter, depending on the presence of functional Sp1 binding sites (33). Thus, further investigation is required to determine whether S100A6 binds to p53 and whether it is involved in the Sp1 binding sites.

In conclusion, the present study revealed that S100A6 was overexpressed in gastric cancer cells, in the cytoplasm and nucleus. Increased expression of S100A6 promoted cell 
proliferation in gastric cancer cells. A promoter ChIP-Chip assay was used to detect the gene promoter regions to which S100A6 may bind directly or indirectly, and 1,328 factors were found, including 57 factors associated with cell proliferation. The overexpression of S100A6 caused a marked increase in the mRNA expression levels of CDK4, CDK5, IL-8, Bcl2 and MCM7. In addition, S100A6 was associated with rRNA transcription. The results suggested that S100A6 may regulate cell proliferation by affecting the expression levels of the above-mentioned factors.

\section{Acknowledgements}

This study was funded by the National Key Technology R\&D Program(grantnos.2012AA02A203-B01,2012AA02A504-B01 and 2012AA020101) and the Beijing Municipal Science \& Technology Commission (grant no. D131100005313010).

\section{References}

1. Cross SS, Hamdy FC, Deloulme JC and Rehman I: Expression of S100 proteins in normal human tissues and common cancers using tissue microarrays: S100A6, S100A8, S100A9 and S100A11 are all overexpressed in common cancers. Histopathology 46: 256-269, 2005 .

2. Wang XH, Zhang LH, Zhong XY, Xing XF, Liu YQ, Niu ZJ, Peng Y, Du H, Zhang GG, Hu Y, et al: S100A6 overexpression is associated with poor prognosis and is epigenetically up-regulated in gastric cancer. Am J Pathol 177: 586-597, 2010.

3. Filipek A and Kuźnicki J: Calcyclin-from basic research to clinical implications. Acta Biochim Pol 40: 321-327, 1993.

4. Shekouh AR, Thompson CC, Prime W, Campbell F, Hamlett J, Herrington CS, Lemoine NR, Crnogorac-Jurcevic T, Buechler MW, Friess H, et al: Application of laser capture microdissection combined with two-dimensional electrophoresis for the discovery of differentially regulated proteins in pancreatic ductal adenocarcinoma. Proteomics 3: 1988-2001, 2003.

5. Brown LM, Helmke SM, Hunsucker SW, Netea-Maier RT, Chiang SA, Heinz DE, Shroyer KR, Duncan MW and Haugen BR Quantitative and qualitative differences in protein expression between papillary thyroid carcinoma and normal thyroid tissue. Mol Carcinog 45: 613-626, 2006.

6. Stulik J, Osterreicher J, Koupilová K, Knízek J, Bures J, Jandík P, Langr F, Dedic K, Schäfer BW and Heizmann CW: Differential expression of the $\mathrm{Ca}_{2}{ }^{+}$binding $\mathrm{S} 100 \mathrm{~A} 6$ protein in normal, preneoplastic and neoplastic colon mucosa. Eur J Cancer 36: 1050-1059, 2000.

7. Komatsu K, Kobune-Fujiwara Y, Andoh A, Ishiguro S, Hunai H, Suzuki N, Kameyama M, Murata K, Miyoshi J, Akedo H, et al: Increased expression of S100A6 at the invading fronts of the primary lesion and liver metastasis in patients with colorectal adenocarcinoma. Br J Cancer 83: 769-774, 2000.

8. Weterman MA, Stoopen GM, van Muijen GN, Kuznicki J, Ruiter DJ and Bloemers HP: Expression of calcyclin in human melanoma cell lines correlates with metastatic behavior in nude mice. Cancer Res 52: 1291-1296, 1992.

9. Pedrocchi M, Schafer BW, Mueller H, Eppenberger U and Heizmann CW: Expression of $\mathrm{Ca}\left(2^{+}\right)$-binding proteins of the S100 family in malignant human breast-cancer cell lines and biopsy samples. Int J Cancer 57: 684-690, 1994.

10. Rehman I, Cross SS, Catto JW, Leiblich A, Mukherjee A, Azzouzi AR, Leung HY and Hamdy FC: Promoter hyper-methylation of calcium binding proteins S100A6 and S100A2 in human prostate cancer. Prostate 65: 322-330, 2005.

11. Rehman I, Cross SS, Azzouzi AR, Catto JW, Deloulme JC, Larre S, Champigneuille J, Fromont G, Cussenot O and Hamdy FC: S100A6 (Calcyclin) is a prostate basal cell marker absent in prostate cancer and its precursors. Br J Cancer 91: 739-744, 2004.
12. Kim J, Kim J, Yoon S, Joo J, Lee Y, Lee K, Chung J and Choe I: S100A6 protein as a marker for differential diagnosis of cholangiocarcinoma from hepatocellular carcinoma. Hepatol Res 23: 274, 2002.

13. Lyu X, Li H, Ma X, Li X, Gao Y, Ni D, Shen D, Gu L, Wang B, Zhang Y and Zhang X: High-level S100A6 promotes metastasis and predicts the outcome of T1-T2 stage in clear cell renal cell carcinoma. Cell Biochem Biophys 71: 279-290, 2015.

14. Emberley ED, Murphy LC and Watson PH: S100 proteins and their influence on pro-survival pathways in cancer. Biochem Cell Biol 82: 508-515, 2004.

15. Golitsina NL, Kordowska J, Wang CL and Lehrer SS: $\mathrm{Ca}_{2}+$-dependent binding of calcyclin to muscle tropomyosin. Biochem Biophys Res Commun 220: 360-365, 1996.

16. Mani RS, McCubbin WD and Kay CM: Calcium-dependent regulation of caldesmon by an 11-kDa smooth muscle calcium-binding protein, caltropin. Biochemistry 31: 11896-11901, 1992.

17. Bao L, Odell AF, Stephen SL, Wheatcroft SB, Walker JH and Ponnambalam S: The S100A6 calcium-binding protein regulates endothelial cell-cycle progression and senescence. FEBS J 279: 4576-4588, 2012.

18. Joo JH, Kim JW, Lee Y, Yoon SY, Kim JH, Paik SG and Choe IS: Involvement of NF-kappaB in the regulation of S100A6 gene expression in human hepatoblastoma cell line HepG2. Biochem Biophys Res Commun 307: 274-280, 2003.

19. Zheng M, Barrera LO, Ren B and Wu YN: ChIP-chip: Data, model, and analysis. Biometrics 63: 787-796, 2007.

20. Vimalachandran D, Greenhalf W, Thompson C, Lüttges J, Prime W,CampbellF, Dodson A, Watson R,Crnogorac-Jurcevic T, Lemoine N, et al: High nuclear S100A6 (Calcyclin) is significantly associated with poor survival in pancreatic cancer patients. Cancer Res 65: 3218-3225, 2005.

21. Livak KJ and Schmittgen TD: Analysis of relative gene expression data using real-time quantitative PCR and the 2(-Delta Delta C(T)) Method. Methods 25: 402-408, 2001.

22. Wu H, Weng D, Weng D, Xing H, Song X, Zhu T, Xia X, Weng Y, Xu G, Meng L, et al: Reversal of the malignant phenotype of ovarian cancer A2780 cells through transfection with wild-type PTEN gene. Cancer Lett 271: 205-214, 2008.

23. Leśniak W, Słomnicki ŁP and Filipek A: S100A6 - new facts and features. Biochem Biophys Res Commun 390: 1087-1092, 2009.

24. Itoh Y, Joh T, Tanida S, Sasaki M, Kataoka H, Itoh K, Oshima T, Ogasawara N, Togawa S, Wada T, et al: IL-8 promotes cell proliferation and migration through metalloproteinase-cleavage proHB-EGF in human colon carcinoma cells. Cytokine 29: 275-282, 2005.

25. Lord JD, McIntosh BC, Greenberg PD and Nelson BH: The IL-2 receptor promotes lymphocyte proliferation and induction of the c-myc, bcl-2, and bcl-x genes through the trans-activation domain of Stat5. J Immunol 164: 2533-2541, 2000.

26. Henriksson JT, Coursey TG, Corry DB, De Paiva CS and Pflugfelder SC: IL-13 stimulates proliferation and expression of mucin and immunomodulatory genes in cultured conjunctival goblet cells. Invest Ophthalmol Vis Sci 56: 4186-4197, 2015.

27. Liu D, Liu C, Wang X, Ingvarsson S and Chen H: MicroRNA-451 suppresses tumor cell growth by down-regulating IL6R gene expression. Cancer Epidemiol 38: 85-92, 2014.

28. Sherr CJ: The Pezcoller lecture: Cancer cell cycles revisited. Cancer Res 60: 3689-3695, 2000.

29. Brinkkoetter PT, Olivier P, Wu JS, Henderson S, Krofft RD, Pippin JW, Hockenbery D, Roberts JM and Shankland SJ: Cyclin I activates $\mathrm{Cdk} 5$ and regulates expression of $\mathrm{Bcl}-2$ and Bcl-XL in postmitotic mouse cells. J Clin Invest 119: 3089-3101, 2009.

30. Padmanabhan V, Callas P, Philips G, Trainer TD and Beatty BG: DNA replication regulation protein $\mathrm{Mcm} 7$ as a marker of proliferation in prostate cancer. J Clin Pathol 57: 1057-1062, 2004.

31. Bouchard C, Staller P and Eilers M: Control of cell proliferation by Myc. Trends Cell Biol 8: 202-206, 1998.

32. Słomnicki ŁP, Nawrot B and Leśniak W: S100A6 binds p53 and affects its activity. Int J Biochem Cell Biol 41: 784-790, 2009.

33. Innocente SA and Lee JM: p53 is a NF-Y- and p21-independent, Sp1-dependent repressor of cyclin B1 transcription. FEBS Lett 579: 1001-1007, 2005. 